\title{
Effects of Intranasal Oxytocin on Emotion Recognition in Korean Male: A Dose-Response Study
}

\author{
Na Young Shin', Hye Yoon Park², Wi Hoon Jung ${ }^{3}$, and Jun Soo Kwon ${ }^{2,4} \bowtie$ \\ ${ }^{1}$ College of Liberal Arts and Interdisciplinary Studies, Kyonggi University, Suwon, Republic of Korea \\ ${ }^{2}$ Department of Psychiatry, Seoul National University College of Medicine, Seoul, Republic of Korea \\ ${ }^{3}$ Department of Psychology, Korea University, Seoul, Republic of Korea \\ ${ }^{4}$ Department of Brain and Cognitive Sciences, College of Natural Sciences, Seoul National University, Seoul, Republic of Korea
}

Objective Research has shown that intranasal oxytocin affects social cognition and behavior; however, its effects vary based on social context, individual characteristics and dose. The present study aimed to determine effective dose of oxytocin spray on emotion recognition in healthy Korean males.

Methods The study followed a randomized, double-blind, placebo-controlled design. Thirty-seven Korean males underwent two experimental sessions, with one week in between. They received either $32(n=19)$ or $40(n=18)$ international units (IU) of oxytocin and placebo, and then completed a face emotion recognition task. The effect of oxytocin on emotion recognition was examined using repeated measures analysis of variance (ANOVA) for each dose condition.

Results The higher dose (40 IU) was found to improve recognition of happy faces, while the lower dose (32 IU) had no effect. There were no statistical differences in age, education, attachment style or empathic ability between the two dose groups.

Conclusion The results suggest that oxytocin increases the ability of Korean males to recognize positive emotion, and this effect is dosedependent. Additional studies evaluating the effect of higher doses of oxytocin on social cognition will help to determine the optimal dose for Korean populations.

Psychiatry Investig 2018;15(7):710-716

Key Words Dose, Emotion, Korean, Intranasal, Oxytocin.

\section{INTRODUCTION}

Throughout the last decade, a number of studies have evaluated the effect of intranasal oxytocin on a variety of social cognitive processes such as trust, emotion recognition, and cooperation. ${ }^{1-4}$ Other studies have explored the effects of intranasal oxytocin on symptoms and social dysfunction in patients with psychological or physical disorders. ${ }^{5-13}$ Evidence from recent studies suggests that the effect of intranasal oxytocin on social cognition varies depending on the social context and individual characteristics. ${ }^{14-16}$ For example, intranasal oxytocin effects may differ based on gender, ${ }^{17-19}$ attachment style, ${ }^{14,20}$

Received: October 31, 2017 Revised: January 30, 2018

Accepted: February 19, 2018

$\triangle$ Correspondence: Jun Soo Kwon, MD, PhD

Department of Psychiatry \& Department of Brain \& Cognitive Sciences, Seoul National University, 101 Daehak-ro, Jongno-gu, Seoul 03080, Republic of Korea Tel: +82-2-2072-2972, Fax: +82-2-747-9063, E-mail: kwonjs@snu.ac.kr

(a) This is an Open Access article distributed under the terms of the Creative Commons Attribution Non-Commercial License (https://creativecommons.org/licenses/bync/4.0) which permits unrestricted non-commercial use, distribution, and reproduction in any medium, provided the original work is properly cited. and in-group/out-group., ${ }^{41}$

Several recent studies have found that the effects of oxytocin are dose-dependent. In a study of healthy German men, oxytocin-induced change in amygdala activity was observed after treatment with 24 international units (IU), but not with the $12 \mathrm{IU}$ dose. ${ }^{22}$ In a sample of healthy Norwegian males, 8 IU oxytocin delivered by a Breath Powered nasal device reduced the perception of angry faces and reduced amygdala activity in response to emotional faces; however, the $24 \mathrm{IU}$ dose was not effective. ${ }^{23,24}$ The dose-dependent effect of oxytocin was also observed in participants with psychiatric symptoms. ${ }^{25,26}$ In a study of patients with autism, symptoms were alleviated after daily treatment with a $32 \mathrm{IU}$ oxytocin spray for 12 weeks, while the 16 IU treatment was not effective. ${ }^{26}$ In a study of subjects at a high risk for psychosis, intranasal oxytocin dose negatively correlated with symptom severity, despite a lack of effect of treatment on social cognition or symptom level. ${ }^{25}$ These results suggest that there may be a minimal and optimal dose for the positive effects on social cognition and behavior, which is likely to be dependent on individual char- 
acteristics. Further research is needed to understand more fully the dose-dependent effects of oxytocin spray on social cognition in individuals with diverse characteristics.

This study was designed to determine the minimal effective dose for intranasal oxytocin to influence emotion recognition in healthy Korean males. Previous studies have reported significant differences between Korean and western populations in terms of attachment styles ${ }^{27}$ and genotype distribution of oxytocin receptor polymorphisms. ${ }^{28,29}$ In studies of social cognitive functions in Korean subjects, oxytocin was found to influence attentional bias to emotional stimuli in patients with eating disorders and in healthy Korean females. ${ }^{30-32}$ Oxytocin can also influence amygdala reactivity during emotion recognition in patients with schizophrenia and in healthy Korean males. ${ }^{8}$ All but one of the above-mentioned studies utilized a dose of 40 IU in female subjects. The present study aimed to determine effective dose of oxytocin spray on emotion recognition in healthy Korean males. In a preliminary small experiment, we observed that a dose of 24 IU had no effect; therefore, we utilized dose of 32 and 40 IU. Based on the results of previous studies, we expected both doses to have a beneficial effect on emotion recognition, with the 40 IU dose having a stronger effect.

\section{METHODS}

\section{Participants}

Thirty-Seven healthy Korean males were recruited through internet advertisements. Subjects were screened for Axis I disorders using the Non-Patient Edition of the Structured Clinical Interview for the Diagnostic and Statistical Manual of Mental Disorders, Fourth Edition (DSM-IV). Subjects were matched by age and separated into two dose groups receiving either $32 \mathrm{IU}(\mathrm{n}=19)$ or $40 \mathrm{IU}(\mathrm{n}=18)$.

The exclusion criteria were mental retardation, brain injury, neurological illness, alcohol or substance abuse, and acetic acid allergy. The study was approved by the Institutional Review Board of Soul National University Hospital (H-1105047-361). After describing the study in detail, written informed consent was obtained from each participant (or a parent if the participant was under 20 years of age).

\section{Measurements}

A face emotion recognition task (FERT) was created using photos of three Korean male and three Korean female faces depicting seven types of facial expressions (happy, fearful, sad, angry, disgust, surprise, and neutral) selected from the ChaeLee Korean Facial Expression of Emotion (C-KFEE) ${ }^{33}$ and Korean Facial Expressions of Emotion (KOFEE) databases. The mean accuracy rates were $99.6 \%$ for happy, $97.5 \%$ for sad, $97.1 \%$ for neutral faces, $88.3 \%$ for surprise, $79.2 \%$ for fearful, and $45 \%$ for disgust based on a sample of 40 Korean university students in our preliminary study. Due to low accuracy in the identification of disgust, this facial expression was excluded from the set. Winmorph 2.0 (http://debugmode. $\mathrm{com} /$ winmorph/) was used to morph the five types of emotional faces with the neutral face of the same person at four intensities $(25,50,75$, and $100 \%)$. Eleven morphed faces were excluded from the set of photographs due to distortion. Each facial expression was randomly presented as the stimulus once for $1 \mathrm{sec}$, followed by a list of the six emotions that remained on the screen until the subject made a decision. Fixation cross hairs were presented for $0.5 \mathrm{sec}$ before each stimulus. The accuracy rate and response time (RT) for correct responses were used in the analysis.

A 10-point visual analog scale (VAS) was used to evaluate the effect of oxytocin on the subjects' mood before and $45 \mathrm{~min}$ after treatment. Subjects were asked to evaluate six parameters (wakefulness, calmness, anger, happiness, sadness, anxiousness).

The Korean version of the Relationship Style Questionnaires (RSQ), ${ }^{34}$ a self-rated 5-point Likert scale, was used to determine attachment style. The 18 items of the RSQ assess four attachment subtypes: secure, preoccupied, dismissing, and fearful. The Korean version of Interpersonal Reactivity Index (IRI), ${ }^{35,36}$ a self-reported 5-point Likert scale, was used to assess multicomponents of empathy: empathic concern, perspective taking, fantasy, and personal distress (28 items).

\section{Drug preparation}

Drug preparation has been described previously. ${ }^{8,37}$ Briefly, $50 \mathrm{~mL}$ of oxytocin (202 IU) was combined with $202.5 \mathrm{~mL}$ of a $0.9 \%$ saline solution $(\mathrm{pH}, 4.02)$. The solution was then distributed in sterilized vials $(3 \mathrm{~mL})$ and stored in the refrigerator. The oxytocin or saline solution (placebo) was then transferred from the vial into a nasal spray device prior to the experiment.

\section{Procedure}

The study followed a randomized, double-blind, placebocontrolled design. According to the procedures of previous studies that found the effects of intranasal oxytocin on emotion recognition, ${ }^{6,38}$ the two treatment sessions were conducted with 1-week interval. The order of drug administration was counterbalanced. Subjects were asked to abstain from alcohol, nicotine, caffeine, and excessive exercise for $24 \mathrm{~h}$ prior to the experiment, and to avoid food and drinks except water for $2 \mathrm{~h}$ prior to the experiment.

After completing the questionnaires, the subjects self-administered 8 (32 IU) or 10 (40 IU) puffs of oxytocin or placebo contained in a nasal spray, switching nostrils under the su- 


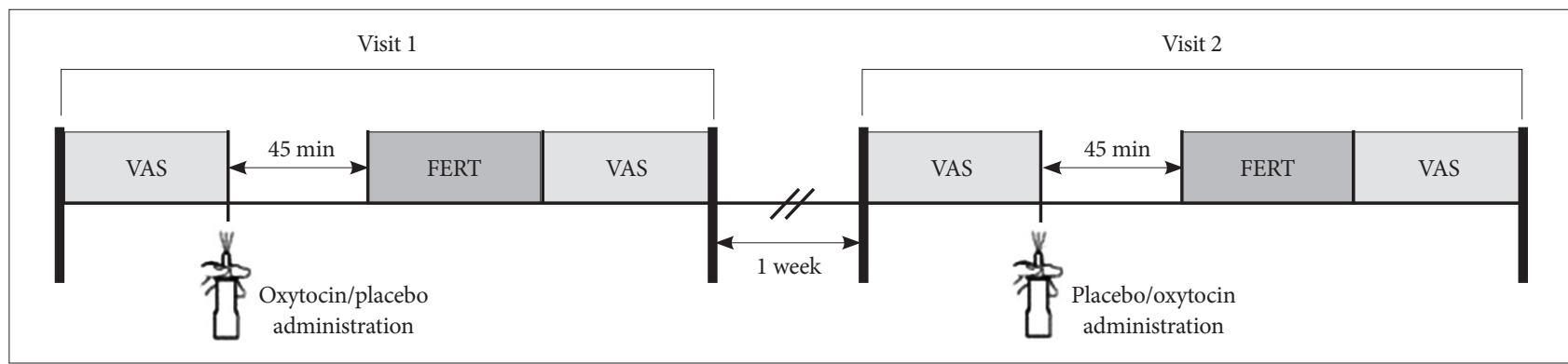

Figure 1. Experimental procedure. The subjects underwent two experimental sessions, with one week in between. The emotion recognition task was performed $45 \mathrm{~min}$ after 8 (32 IU) or 10 (40 IU) puffs of oxytocin or placebo. VAS: visual analog scale, FERT: face emotion recognition task.

pervision of an experimenter. The task was performed 45 min after drug administration (Figure 1). The experiment was carried out first with the subjects assigned to the $32 \mathrm{IU}$ dose condition, followed by the subjects to the 40 IU dose condition. The subjects given the 40 IU dose of oxytocin performed the same task prior drug treatment to examine the influence of baseline performance on the efficacy of oxytocin.

\section{Statistical analysis}

An independent t-test was used to compare age and education levels between subjects in the two dose groups. Multivariate analysis of variance (MANOVA) was performed to examine psychological characteristics. The effect of oxytocin on emotion recognition or mood changes (pre-treatment scorepost-treatment score) was examined using repeated measures ANOVA with treatment (oxytocin, placebo) and the task or mood variables as within-subject factors. Pearson correlation analysis was conducted to evaluate the relationship between psychological characteristics and the effect of oxytocin on emotion recognition. The analyses were performed using the IBM Statistical Package for the Social Sciences version 22 (IBM Corp., Armonk, NY, USA).

\section{RESULTS}

\section{Demographical and psychological characteristics}

There was no significant difference in age $\left(\mathrm{t}_{35}=-0.33, \mathrm{p}=0.74\right)$ or education level $\left(\mathrm{t}_{35}=0.70, \mathrm{p}=0.49\right)$ between the two groups (Table 1). Similarly, no differences were found with RSQ $\left(\mathrm{F}_{4,32}=\right.$ $0.89, \mathrm{p}=0.48)$ or IRI scores $\left(\mathrm{F}_{4,32}=1.47, \mathrm{p}=0.24\right)$ (Table 1$)$.

\section{Effects of oxytocin on facial emotion recognition after 32 IU treatment}

For the accuracy rate of the FERT, a 2 (drug) $\times 6$ (emotional valence) analysis showed a significant main effect of emotional valence $\left(\mathrm{F}_{5,14}=43.6, \mathrm{p}<0.001, \eta_{\mathrm{p}}{ }^{2}=0.94\right)$, indicating the highest accurate rates for neutral $(93.4 \pm 8.1)$ faces, followed by happy (83.0 $\pm 6.3 \%)$, angry $(80.3 \pm 6.4 \%)$, sad $(76.4 \pm 6.3 \%)$, sur-
Table 1. Psychological scale scores in subjects

\begin{tabular}{lcclc}
\hline & $\begin{array}{c}32 \mathrm{IU} \\
(\mathrm{N}=19)\end{array}$ & $\begin{array}{c}40 \mathrm{IU} \\
(\mathrm{N}=18)\end{array}$ & Statistics & $\mathrm{p}$ \\
\hline Age (year) & $22.8(3.2)$ & $23.1(2.8)$ & $\mathrm{t}=-0.33$ & 0.74 \\
Education years & $13.9(1.4)$ & $13.6(1.5)$ & $\mathrm{t}=0.70$ & 0.49 \\
RSQ & & & $\mathrm{F}=0.89$ & 0.48 \\
$\quad$ Secure & $18.8(3.8)$ & $18.6(3.0)$ & & \\
$\quad$ Preoccupied & $12.1(1.9)$ & $12.7(2.1)$ & & \\
$\quad$ Dismissing & $12.5(1.3)$ & $11.8(1.9)$ & & \\
$\quad$ Fearful & $8.2(1.9)$ & $8.6(2.2)$ & & \\
IRI & & & $\mathrm{F}=1.47$ & 0.24 \\
$\quad$ Empathic concern & $23.3(4.0)$ & $26.2(4.6)$ & & \\
Perspective taking & $25.1(5.2)$ & $25.2(4.5)$ & & \\
Fantasy & $25.3(4.1)$ & $26.4(3.9)$ & & \\
Personal distress & $17.5(4.3)$ & $19.6(4.9)$ & & \\
\hline
\end{tabular}

Values are mean with standard deviation in parenthesis. IU: international unit, RSQ: Relationship Style Questionnaire, IRI: Interpersonal Reactivity Index

prised $(66.2 \pm 6.2 \%)$, and fearful faces $(53.3 \pm 13.5 \%)$. No main effect of drug $\left(\mathrm{F}_{1,18}=2.07, \mathrm{p}=0.17, \eta_{\mathrm{p}}{ }^{2}=0.10\right)$ or interaction effect $\left(\mathrm{F}_{5,14}=0.34, \mathrm{p}=0.88, \eta_{\mathrm{p}}{ }^{2}=0.11\right)$ was significant for accuracy. For RT, emotional valence had a significant main effect $\left(\mathrm{F}_{5,14}=\right.$ $13.19, \mathrm{p}<0.001, \eta_{\mathrm{p}}{ }^{2}=0.83$ ), with the longest RT for angry faces $(1.56 \pm 0.84 \mathrm{sec})$ and the shortest for neutral faces $(0.65 \pm 0.24$ sec) (Table 2, Figure 2). No main effect of drug $\left(\mathrm{F}_{1,18}=2.46, \mathrm{p}=\right.$ $\left.0.14, \eta_{\mathrm{p}}{ }^{2}=0.12\right)$ or interaction effect $\left(\mathrm{F}_{5,14}=1.05, \mathrm{p}=0.43, \eta_{\mathrm{p}}{ }^{2}=\right.$ 0.27 ) was significant for RT. No main or interaction effect was observed for mood change score, as measured by subtracting pre-treatment from post-treatment VAS scores.

\section{Effect of oxytocin on facial emotion recognition after 40 IU treatment}

For the accuracy rate of the FERT, a 2 (drug) $\times 6$ (emotional valence) analysis yielded a significant interaction of drug $\times$ emotional valence $\left(\mathrm{F}_{5,13}=4.54, \mathrm{p}=0.01, \eta_{\mathrm{p}}{ }^{2}=0.64\right)$. A post-hoc paired t-test showed significant improvement in recognizing 
the happy faces after oxytocin treatment compared to placebo $\left(t_{17}=3.22, p=0.005\right)$, whereas there was no significant change in differentiating other types of emotional faces after oxytocin administration (Table 2, Figure 2). A main effect of emotional valence was also significant $\left(\mathrm{F}_{5,13}=38.6, \mathrm{p}<0.001, \eta_{\mathrm{p}}{ }^{2}=0.94\right)$, indicating the highest accuracy rates for neutral faces $(87.7 \pm$ $14.3 \%)$, followed by expressions of happiness ( $83.3 \pm 8.2 \%)$, anger $(82.3 \pm 5.2 \%)$, sadness $(75.8 \pm 7.5 \%)$, surprise $(68.0 \pm 11.0 \%)$, and fear $(50.0 \pm 15.0 \%)$. There was no significant main effect of drug for accuracy $\left(\mathrm{F}_{1,17}=0.25, \mathrm{p}=0.62, \eta_{\mathrm{p}}{ }^{2}=0.02\right)$. For RT, a

Table 2. Performance in the face emotion recognition task in each drug-dose condition

\begin{tabular}{|c|c|c|c|c|c|c|}
\hline & \multicolumn{2}{|c|}{$32 \mathrm{IU}(\mathrm{N}=19)$} & \multicolumn{4}{|c|}{$40 \mathrm{IU}(\mathrm{N}=18)$} \\
\hline & Placebo & Oxytocin & Placebo base & Placebo & Oxytocin base & Oxytocin \\
\hline \multicolumn{7}{|c|}{ Accuracy (\%) } \\
\hline Happy & $83.9(6.4)$ & $82.2(8.4)$ & $84.3(11.3)$ & $80.2(9.8)$ & $80.1(8.1)$ & $86.1(8.8)$ \\
\hline Fearful & $53.7(16.5)$ & $52.9(14.2)$ & $48.8(12.8)$ & $49.8(18.4)$ & $47.3(12.8)$ & $49.1(16.1)$ \\
\hline Surprise & $66.2(9.4)$ & $66.2(7.7)$ & $71.8(10.4)$ & $67.6(13.4)$ & $67.9(15.1)$ & $68.8(11.3)$ \\
\hline Angry & $81.6(5.8)$ & $78.9(8.6)$ & $81.0(5.8)$ & $82.3(5.6)$ & $80.2(6.2)$ & $82.8(5.9)$ \\
\hline Sad & $78.2(8.3)$ & $74.7(6.4)$ & $75.4(11.6)$ & $77.0(8.5)$ & $67.9(15.1)$ & $75.4(7.5)$ \\
\hline Neutral & $93.9(10.0)$ & $93.0(10.1)$ & $82.4(16.1)$ & $90.2(13.3)$ & $83.3(17.1)$ & $83.3(19.0)$ \\
\hline \multicolumn{7}{|l|}{ RT (sec) } \\
\hline Happy & $0.71(0.29)$ & $0.67(0.24)$ & $0.88(0.52)$ & $0.58(0.33)$ & $0.85(0.47)$ & $0.54(0.40)$ \\
\hline Fearful & $1.60(1.00)$ & $1.46(0.90)$ & $1.19(0.71)$ & $0.75(0.47)$ & $1.21(0.57)$ & $0.86(0.49)$ \\
\hline Surprise & $1.25(0.54)$ & $1.05(0.37)$ & $1.06(0.64)$ & $0.70(0.33)$ & $1.08(0.40)$ & $0.78(0.38)$ \\
\hline Angry & $1.08(0.31)$ & $1.01(0.41)$ & $1.23(0.46)$ & $0.75(0.31)$ & $1.04(0.37)$ & $0.83(0.38)$ \\
\hline Sad & $1.18(0.45)$ & $0.97(0.41)$ & $0.96(0.34)$ & $0.80(0.29)$ & $1.00(0.36)$ & $0.82(0.33)$ \\
\hline Neutral & $0.71(0.07)$ & $0.57(0.20)$ & $0.80(0.44)$ & $0.56(0.29)$ & $0.98(0.51)$ & $0.58(0.36)$ \\
\hline \multicolumn{7}{|l|}{$\mathrm{VAS}_{\text {change }}$} \\
\hline Wakeful & $-1.95(12.47)$ & $-0.79(11.77)$ & & 4.94 (11.77) & & $3.89(9.23)$ \\
\hline Calm & $2.00(8.74)$ & $1.63(17.75)$ & & $-3.78(7.44)$ & & $-1.11(15.40)$ \\
\hline Happy & $-2.42(6.91)$ & $0.16(11.35)$ & & $1.06(10.79)$ & & $-1.17(12.05)$ \\
\hline Sad & $6.79(10.66)$ & $7.00(10.05)$ & & 2.67 (10.64) & & $0.94(10.41)$ \\
\hline Anxious & $3.68(17.08)$ & $0.16(14.93)$ & & $8.00(11.29)$ & & $4.56(20.82)$ \\
\hline Angry & $-0.63(2.31)$ & $4.00(8.19)$ & & $4.72(8.82)$ & & $-0.67(5.73)$ \\
\hline
\end{tabular}

Values are mean with standard deviation in parenthesis. IU: international unit, Placebo base: performance before placebo treatment, Oxytocin base: performance before oxytocin treatment, RT: reaction time, $\mathrm{VAS}_{\text {change: }}$ pre-treatment score-post-treatment score of visual analog scale

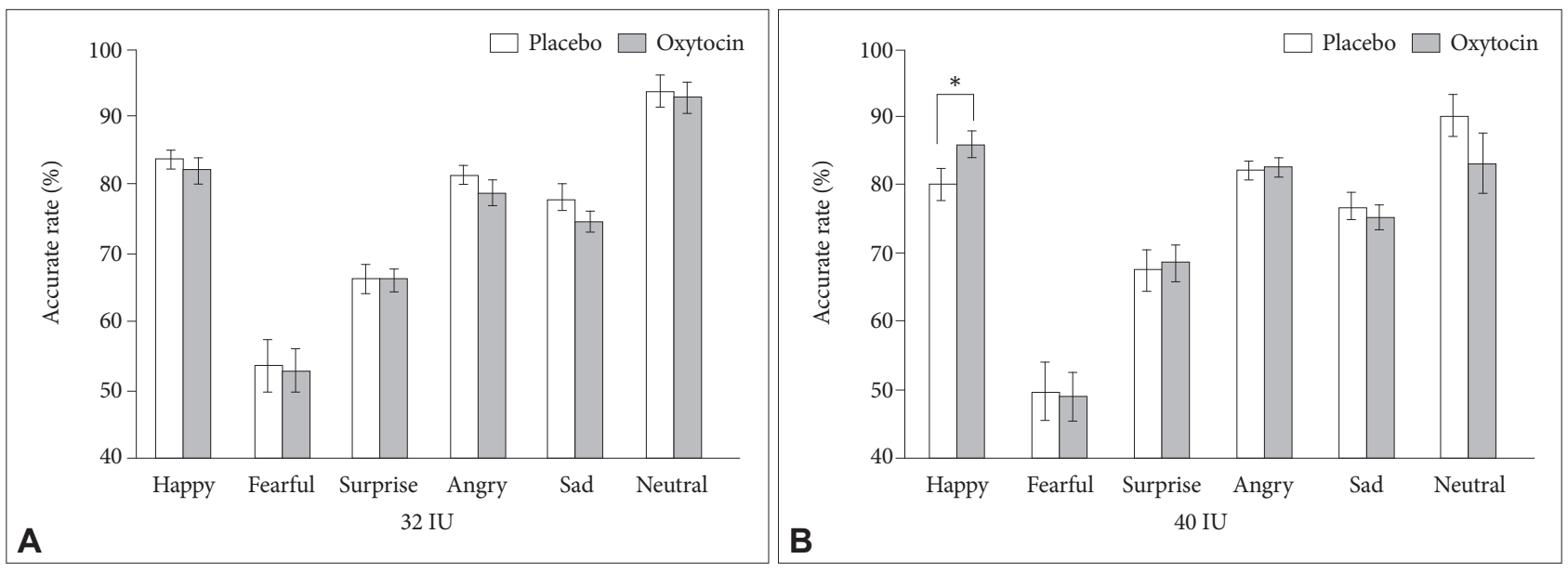

Figure 2. Mean Accuracy in the Face Emotion Recognition Task After $32 \mathrm{IU}$ and 40 IU Treatment. A: Mean accuracy to recognize emotional faces after $32 \mathrm{IU}$ treatment, B: Mean accuracy to recognize emotional faces after $40 \mathrm{IU}$ treatment. *Indicates a significant difference in performance between oxytocin and placebo $(p<0.005)$. IU: international unit; Error bars indicate standard error of mean. 
main effect of emotional valence was only significant $\left(\mathrm{F}_{5,13}=\right.$ 5.21, $\mathrm{p}=0.008, \eta_{\mathrm{p}}{ }^{2}=0.67$ ), indicating the longest RT for fear $(0.81 \pm 0.37 \mathrm{sec})$ and the shortest for happy faces $(0.56 \pm 0.31$ sec). No main effect of drug $\left(\mathrm{F}_{1,17}=0.08, \mathrm{p}=0.78, \eta_{\mathrm{p}}{ }^{2}=0.01\right)$ or interaction effect $\left(\mathrm{F}_{5,13}=0.47, \mathrm{p}=0.79, \eta_{\mathrm{p}}{ }^{2}=0.15\right)$ was significant for RT.

To examine whether the effect of oxytocin would remain after adjusting pretreatment performance of the FERT, a change ratio was calculated by subtracting post-treatment score from the pre-treatment score and dividing by the pretreatment score for each drug condition. A 2 (drug) $\times 6$ (emotional valance) analysis revealed a significant interaction effect of drug $\times$ emotional valence for the change ratio of accuracy $\left(\mathrm{F}_{5,12}=5.04, \mathrm{p}=0.010, \eta_{\mathrm{p}}{ }^{2}=0.68\right)$. A post-hoc paired t-test showed a significant increase in the change ratio only for happy faces after oxytocin treatment compared to the placebo treatment ( $8.41 \pm 7.2 \%$ vs. $-3.81 \pm 7.97 \%)$. No other main or interaction effects were significant for accuracy or RT.

\section{Correlation between psychological variables and oxytocin effects}

For the correlation analysis, percentage change was calculated by subtracting performance on the FERT in the placebo condition from performance in the oxytocin condition. No significant correlation between percentage changes and the RSQ or KIRI was found with the 32 or 40 IU dose groups after Bonferroni correction $(\mathrm{p}<0.003)$.

\section{DISCUSSION}

This study investigated the effects of intranasal oxytocin on emotion recognition in health Korean males for two dose conditions (32 and $40 \mathrm{IU}$ ). Subjects' enhanced recognition of happy facial expressions was observed with the 40 IU treatment, but not the 32 IU treatment. The improved recognition of happy faces after the $40 \mathrm{IU}$ treatment remained after adjusting for baseline performance. These finding are consistent with earlier studies reporting oxytocin-related effects in Korean subjects after $40 \mathrm{IU}$ treatment. ${ }^{8,30-32}$ This study is the first to show an insignificant effect of oxytocin on emotion recognition at a dose of less than $40 \mathrm{IU}$ in Korean males. These results confirm earlier studies reporting a dose-dependent effect of intranasal oxytocin on social cognition.

In this study, the beneficial effect of oxytocin was found only for the happy faces after $40 \mathrm{IU}$ treatment. This result corresponds to our previous fMRI study that showed increased amygdala activity during emotion recognition after oxytocin treatment of $40 \mathrm{IU}$ in Korean healthy male, with the effect being most apparent for the happy faces. ${ }^{8}$ Our findings are also consistent with previous studies that showed improved recognition of positive emotion after oxytocin administration in healthy persons, ${ }^{37-39}$ but inconsistent with results from a recent meta-analysis that showed the effect of oxytocin on the recognition of fear. ${ }^{2}$ The specific effect in relation to happy faces in the current study might be attributed to the distinct cultural features of Korean people with regard to emotion recognition. Multiple studies suggest that Asians, including Koreans, have different views about positive and negative emotions compared to Westerners. For example, Koreans tend to recognize positive emotions more negatively and negative emotions more positively compared to Western people. ${ }^{40}$ Moreover, people from interdependent cultures, such as Koreans and Chinese, value lower, more than high-arousal, positive expression. ${ }^{41,42}$ Therefore, it is plausible that inhaled oxytocin changes negativity about the positive state in Koreans by increasing the social approach motivation. It is known that oxytocin spray increases pro-sociality by improving affiliate behaviors such as expression of positive emotion, trust, cooperation, and altruism. ${ }^{2,15}$ It is possible that intranasal oxytocin plays a role in enhancing perceptive sensitivity by increasing affiliative motivation to positive affective state in healthy Korean males.

The 32 IU oxytocin dose had no effect on emotion recognition in Korean males. This is inconsistent with many previous studies that have found oxytocin-induced effects on emotion recognition after smaller dose treatment in healthy people. ${ }^{22,37,38,43-46}$ The lack of efficacy of 32 IU oxytocin is probably not due to the demographical and psychological characteristics of participants, since there were no differences between the participants in $32 \mathrm{IU}$ and $40 \mathrm{IU}$ dose conditions with regard to age, level of education, empathy, ability, and attachment style. Recent studies have shown that oxytocin spray was effective at improving social cognitive function in certain doses but not in other doses in both healthy persons and patients with mental disorders. ${ }^{22,24,26,47}$ Further, one study found that an increased dose of oxytocin was associated with a better effect, ${ }^{25}$ whereas the other study revealed that a high dose of oxytocin (48 IU) had a reverse effect. ${ }^{22}$ Our results correspond with those of previous findings indicating the dose-dependent effect of intranasal oxytocin in Korean males.

The effect of oxytocin observed after the 40 IU treatment, but not after $32 \mathrm{IU}$, suggests that at least 5 puffs in each nostril may be required to obtain a positive effect in Korean males. Oxytocin-related effects on emotion recognition have been found at doses of 20 to $40 \mathrm{IU}^{2}$ Although the mechanism underlying oxytocin-induced effect and brain- delivery routes of intranasal oxytocin in human is not clear, evidence increasingly indicates that oxytocin efficacy depends on various factors such as oxytocin receptor genotype, ${ }^{26,48}$ attachment style, ${ }^{20}$ early life stress ${ }^{43}$ and thinking style. ${ }^{49}$ From these findings, specific properties of oxytocin receptor genotyp $\mathrm{e}^{28}$ and attachment 
style ${ }^{27}$ in Koreans may help to determine the effective dose of oxytocin spray. Alternatively, low emotional arousal in Koreans may influence oxytocin efficacy. Cross-cultural studies have reported that Asians have lower emotional arousal level than Western populations. ${ }^{50}$ as they perceive facial expressions to have a lower intensity ${ }^{51}$ and express less emotion. ${ }^{52}$ The psychological and biological characteristics of Koreans may have an impact on the threshold for oxytocin-related changes in emotional processing. Further studies are needed to investigate the effect of oxytocin at doses above 40 IU to determine an optimal dose for Korean subjects.

This study has several limitations. First, the study utilized a between-subjects design in which the participants were treated with 32 or $40 \mathrm{IU}$ oxytocin. Although there was no statistical difference between the two dose groups in the characteristics we measured, some differences in unmeasured properties may have affected the results. Secondly, the sample size of each dose condition was small. The lack of oxytocin effect in 32 IU dose condition might be due to a small sample size, implying low statistical power. The effect size of interaction effect for RT was large in $32 \mathrm{IU}$ dose condition. This would suggest that the effect of oxytocin may be found in response time for specific emotional faces after 32 IU treatment at a larger sample. Further studies are needed to investigate the effects of oxytocin on emotion recognition at doses lower than $40 \mathrm{IU}$ in larger Korean samples. Third, the participants in this study were all male. It has been reported that intranasal oxytocin may act differently in men and women. ${ }^{17-19}$ Although some studies observed a beneficial effect on social cognition in Korean females after 40 IU treatment, ${ }^{30,32}$ no study has investigated the efficacy of oxytocin at doses lower than 40 IU.

In conclusion, $40 \mathrm{IU}$ intranasal oxytocin was found to improve recognition of happy faces in Korean males. The $40 \mathrm{IU}$ dose may increase sensitivity to positive emotion in Korean subjects. Our findings suggest that the amount of inhaled oxytocin is an important factor influencing oxytocin efficacy, and the effective dose varies based on individual characteristics. Further studies are required determine the effect of doses higher than $40 \mathrm{IU}$, in individuals with different characteristics including patients in clinical settings.

\section{Acknowledgments}

This study has funded by Basic Science Research Program through the National Research Foundation of Korea (NRF) funded by the Ministry of Science, ICT and Future Planning (2016R1E1A1A02921618).

\section{REFERENCES}

1. Kosfeld M, Heinrichs M, Zak P, Fischbacher U, Fehr E. Oxytocin increases trust in humans. Nature 2005;435:673-676.

2. Leppanen J, Ng KW, Tchanturia K, Treasure J. Meta-analysis of the effects of intranasal oxytocin on interpretation and expression of emotions. Neurosci Biobehav Rev 2017;78:125-144.
3. Chen X, Gautam P, Haroon E, Rilling JK. Within vs. between-subject effects of intranasal oxytocin on the neural response to cooperative and non-cooperative social interactions. Psychoneuroendocrinology 2017; 78:22-30.

4. Ten Velden FS, Daughters K, De Dreu CKW. Oxytocin promotes intuitive rather than deliberated cooperation with the in-group. Horm Behav 2017;92:164-171.

5. Mitchell IJ, Gillespie SM, Abu-Akel A. Similar effects of intranasal oxytocin administration and acute alcohol consumption on socio-cognitions, emotions and behaviour: implications for the mechanisms of action. Neurosci Biobehav Rev 2015;55:98-106.

6. Labuschagne I, Phan KL, Wood A, Angstadt M, Chua P, Heinrichs M, et al. Oxytocin attenuates amygdala reactivity to fear in generalized social anxiety disorder. Neuropsychopharmacology 2010;35:2403-2413.

7. Williams DR, Burkner PC. Effects of intranasal oxytocin on symptoms of schizophrenia: a multivariate Bayesian meta-analysis. Psychoneuroendocrinology 2017;75:141-151.

8. Shin NY, Park HY, Jung WH, Park JW, Yun JY, Jang JH, et al. Effects of oxytocin on neural response to facial expressions in patients with schizophrenia. Neuropsychopharmacology 2015;40:1919-1927.

9. Preti A, Melis M, Siddi S, Vellante M, Doneddu G, Fadda R. Oxytocin and autism: a systematic review of randomized controlled trials. J Child Adolesc Psychopharmacol 2014;24:54-68.

10. van Zuiden M, Frijling JL, Nawijn L, Koch SBJ, Goslings JC, Luitse JS, et al. Intranasal oxytocin to prevent posttraumatic stress disorder symptoms: a randomized controlled trial in emergency department patients. Biol Psychiatry 2017;81:1030-1040.

11. Rash JA, Toivonen K, Robert M, Nasr-Esfahani M, Jarrell JF, Campbell TS. Protocol for a placebo-controlled, within-participants crossover trial evaluating the efficacy of intranasal oxytocin to improve pain and function among women with chronic pelvic musculoskeletal pain. BMJ Open 2017;7:e014909.

12. Gallup AC, Church AM. The effects of intranasal oxytocin on contagious yawning. Neurosci Lett 2015;607:13-16.

13. Graustella AJ, MacLeod C. A critical review of the influence of oxytocin nasal spray on social cognition in humans: Evidence and future directions. Horm Behav 2012;61:410-418.

14. Szymanska M, Schneider M, Chateau-Smith C, Nezelof S, Vulliez-Coady L. Psychophysiological effects of oxytocin on parent-child interactions: A literature review on oxytocin and parent-child interactions. Psychiatry Clin Neurosci 2017;71:690-705.

15. Bartz JA, Zaki J, Bolger N, Ochsner KN. Social effects of oxytocin in humans: context and person matter. Trends Cogn Sci 2011;15:301-309.

16. Bakermans-Kranenburg MJ, van Ijzendoorn MH. Sniffing around oxytocin: review and meta-analyses of trials in healthy and clinical groups with implications for pharmacotherapy. Transl Psychiatry 2013;3:e258.

17. Rilling JK, Demarco AC, Hackett PD, Chen X, Gautam P, Stair S, et al. Sex differences in the neural and behavioral response to intranasal oxytocin and vasopressin during human social interaction. Psychoneuroendocrinology 2014;39:237-248.

18. Scheele D, Striepens N, Kendrick KM, Schwering C, Noelle J, Wille A, et al. Opposing effects of oxytocin on moral judgment in males and females. Hum Brain Mapp 2014;35:6067-6076.

19. Feng C, Hackett PD, DeMarco AC, Chen X, Stair S, Haroon E, et al. Oxytocin and vasopressin effects on the neural response to social cooperation are modulated by sex in humans. Brain Imaging Behav 2015;9: 754-764.

20. Bartz JA, Lydon JE, Kolevzon A, Zaki J, Hollander E, Ludwig N, et al. Differential effects of oxytocin on agency and communion for anxiously and avoidantly attached individuals. Psychol Sci 2015;26:1177-1186.

21. Ma X, Luo L, Geng Y, Zhao W, Zhang Q, Kendrick KM. Oxytocin increases liking for a country's people and national flag but not for other cultural symbols or consumer products. Front Behav Neurosci 2014;8: 266.

22. Spengler FB, Schultz J, Scheele D, Essel M, Maier W, Heinrichs M, et al. 
Kinetics and dose dependency of intranasal oxytocin effects on amygdala reactivity. Biol Psychiatry 2017;82:885-894.

23. Quintana DS, Westlye LT, Alnaes D, Rustan OG, Kaufmann T, Smerud KT, et al. Low dose intranasal oxytocin delivered with Breath Powered device dampens amygdala response to emotional stimuli: A peripheral effect-controlled within-subjects randomized dose-response fMRI trial. Psychoneuroendocrinology 2016;69:180-188.

24. Quintana DS, Westlye LT, Rustan OG, Tesli N, Poppy CL, Smevik H, et al. Low-dose oxytocin delivered intranasally with Breath Powered device affects social-cognitive behavior: a randomized four-way crossover trial with nasal cavity dimension assessment. Transl Psychiatry 2015;5:e602.

25. Cacciotti-Saija C, Langdon R, Ward PB, Hickie IB, Scott EM, Naismith SL, et al. A double-blind randomized controlled trial of oxytocin nasal spray and social cognition training for young people with early psychosis. Schizophr Bull 2015;41:483-493.

26. Kosaka H, Okamoto Y, Munesue T, Yamasue H, Inohara K, Fujioka T, et al. Oxytocin efficacy is modulated by dosage and oxytocin receptor genotype in young adults with high-functioning autism: a 24-week randomized clinical trial. Transl Psychiatry 2016;6:e872.

27. You HS, Malley-Morrison K. Young adult attachment styles and intimate relationships with close friends: A cross-cultural study of Koreans and Caucasian Americans. J Cross Cult Psychol 2000;31:528-534.

28. Kim HS, Sherman DK, Mojaverian T, Sasaki JY, Park J, Suh EM, et al. Gene-culture interaction: oxytocin receptor polymorphism (OXTR) and emotion regulation. Soc Psychol Pers Sci 2011;2:665-672.

29. Sasaki JY, Kim HS, Xu J. Religion and well-being: the moderating role of culture and the oxytocin receptor (OXTR) gene. J Cross Cult Psychol 2011;42:1394-1405.

30. Kim YR, Eom JS, Yang JW, Kang J, Treasure J. The impact of oxytocin on food intake and emotion recognition in patients with eating disorders: a double blind single dose within-subject cross-over design. PLoS One 2015;10:e0137514.

31. Kim YR, Kim CH, Park JH, Pyo J, Treasure J. The impact of intranasal oxytocin on attention to social emotional stimuli in patients with anorexia nervosa: a double blind within-subject cross-over experiment. PLoS One 2014;9:e90721.

32. Kim YR, Oh SM, Corfield F, Jeong DW, Jang EY, Treasure J. Intranasal oxytocin lessens the attentional bias to adult negative faces: a double blind within-subject experiment. Psychiatry Investig 2014;11:160-166.

33. Lee KU, Kim J, Yeon B, Kim SH, Chae JH. Development and standardization of extended ChaeLee Korean Facial Expressions of Emotions. Psychiatry Investig 2013;10:155-163.

34. Griffin D, Bartholomew K. Models of the self and other: fundamental dimensions underlying measures of adult attachment. J Pers Soc Psychol 1994;67:430-445.

35. Kang I, Kee SW, Kim SE, Jeong B, Hwang JH, Song JE, et al. Reliability and validity of the Korean version of Interpersonal Reactivity Index. J Korean Neuropsychiatr Assoc 2009;48:352-358.

36. Davis MH. Measuring individual differences in empathy: evidence for a multidimensional approach. J Pers Soc Psychol 1983;44:113-126.
37. Marsh AA, Yu HH, Pine DS, Blair RJ. Oxytocin improves specific recognition of positive facial expressions. Psychopharmacology (Berl) 2010;209:225-232.

38. Gamer M, Zurowski B, Buchel C. Different amygdala subregions mediate valence-related and attentional effects of oxytocin in humans. Proc Natl Acad Sci U S A 2010;107:9400-9405.

39. Di Simplicio M, Massey-Chase R, Cowen P, Harmer C. Oxytocin enhances processing of positive versus negative emotional information in healthy male volunteers. J Psychopharmacol 2009;23:241-248.

40. An S, Ji LJ, Marks M, Zhang Z. Two sides of emotion: exploring positivity and negativity in six basic emotions across cultures. Front Psychol 2017;8:610

41. Park B, Tsai JL, Chim L, Blevins E, Knutson B. Neural evidence for cultural differences in the valuation of positive facial expressions. Soc Cogn Affect Neurosci 2016;11:243-252.

42. Tsai JL, Knutson B, Fung HH. Cultural variation in affect valuation. J Pers Soc Psychol 2006;90:288-307.

43. Feeser M, Fan Y, Weigand A, Hahn A, Gartner M, Aust S, et al. The beneficial effect of oxytocin on avoidance-related facial emotion recognition depends on early life stress experience. Psychopharmacology (Berl) 2014;231:4735-4744.

44. Fischer-Shofty M, Shamay-Tsoory SG, Harari H, Levkovitz Y. The effect of intranasal administration of oxytocin on fear recognition. Neuropsychologia 2010;48:179-184.

45. Kirkpatrick MG, Lee R, Wardle MC, Jacob S, de Wit H. Effects of MDMA and intranasal oxytocin on social and emotional processing. Neuropsychopharmacology 2014;39:1654-1663.

46. Lischke A, Berger C, Prehn K, Heinrichs M, Herpertz SC, Domes G. Intranasal oxytocin enhances emotion recognition from dynamic facial expressions and leaves eye-gaze unaffected. Psychoneuroendocrinology 2012;37:475-481.

47. Quintana DS, Westlye LT, Hope S, Naerland T, Elvsashagen T, Dorum $\mathrm{E}$, et al. Dose-dependent social-cognitive effects of intranasal oxytocin delivered with novel Breath Powered device in adults with autism spectrum disorder: a randomized placebo-controlled double-blind crossover trial. Transl Psychiatry 2017;7:e1136.

48. Feng C, Lori A, Waldman ID, Binder EB, Haroon E, Rilling JK. A common oxytocin receptor gene (OXTR) polymorphism modulates intranasal oxytocin effects on the neural response to social cooperation in humans. Genes Brain Behav 2015;14:516-525.

49. Ma Y, Liu Y, Rand DG, Heatherton TF, Han S. Opposing Oxytocin Effects on Intergroup Cooperative Behavior in Intuitive and Reflective Minds. Neuropsychopharmacology 2015;40:2379-2387.

50. Lim N. Cultural differences in emotion: differences in emotional arousal level between the East and the West. Integr Med Res 2016;5:105-109.

51. Matsumoto D, Ekman P. American-Japanese cultural differences in intensity ratings of facial expressions of emotion. Motiv Emot 1989;13: 143-157.

52. Matsumoto D, Kudoh T, Scherer K, Wallbott H. Antecedents of and reactions to emotions in the United States and Japan. J Cross Cult Psychol 1988;19:267-286. 